\title{
Visfatin attenuates the ox-LDL-induced senescence of endothelial progenitor cells by upregulating SIRT1 expression through the PI3K/Akt/ERK pathway
}

\author{
GUANG-FENG MING ${ }^{1,4}$, YONG-JUN TANG ${ }^{2}, \mathrm{KAI} \mathrm{HU}^{3}$, YAO CHEN $^{2}$, WEI-HUA HUANG ${ }^{2}$ and JIAN XIAO ${ }^{1}$ \\ Departments of ${ }^{1}$ Pharmacy, ${ }^{2}$ Clinical Pharmacology, ${ }^{3}$ Neurology and ${ }^{4}$ Critical Care Medicine, \\ Xiangya Hospital, Central South University, Changsha, Hunan 410008, P.R. China
}

Received September 1, 2015; Accepted May 26, 2016

DOI: $10.3892 /$ ijmm.2016.2633

\begin{abstract}
Endothelial progenitor cells (EPCs) play an important role in aging-associated senescence, thereby potentially contributing to vascular pathologies. Visfatin, identified as a new adipocytokine, is closely associated with the senescence of human cells. However, the effects of visfatin on the oxidized low-density lipoprotein (ox-LDL)-induced senescence of EPCs has not yet been explored, to the best of our knowledge. For this purpose, in the present study, we examined the effects of visfatin in ox-LDL-stimulated EPCs as well as the underlying mechanism responsible for these effects. We found that visfatin attenuated the ox-LDL-induced senescence of EPCs by repressing $\beta$-galactosidase expression and recovering telomerase activity. Western blot analysis confirmed that visfatin induced a dose-dependent increase in sirtuin 1 (SIRT1) expression in EPCs and ox-LDL exposure decreased SIRT1 expression. Silencing SIRT1 abolished the inhibition of EPC senescence and the suppression of p53 expression induced by visfatin. Moreover, visfatin attenuated the inhibition of phosphorylation of Akt, phosphoinositide-3-kinase (PI3K) and extracellular signal-regulated kinase (ERK) induced by ox-LDL. Taken together, these findings suggest that the treatment of EPCs with visfatin markedly attenuates the ox-LDL-induced senescence of EPCs by upregulating SIRT1 expression through the PI3K/Akt/ERK pathway.
\end{abstract}

\section{Introduction}

Endothelial progenitor cells (EPCs) play an important role in vascular morphogenesis, both during embryonic development and in postnatal neovascularization, thereby contributing to tissue maintenance and repair in various pathological conditions $(1,2)$.

Correspondence to: Dr Jian Xiao, Department of Pharmacy, Xiangya Hospital, Central South University, 110 Xiangya Road, Changsha, Hunan 410008, P.R. China

E-mail: jxiao2578@163.com

Key words: visfatin, sirtuin 1, senescence, endothelial progenitor cells, PI3K/Akt/ERK pathway
Previous findings have shown that EPCs contribute to blood flow recovery in ischemic limbs and improve left ventricular function following myocardial infarction $(3,4)$, indicating a potential role for EPCs in the cell-based therapeutic neovascularization of ischemic tissues. However, the numbers and function of EPCs decline as part of aging-associated senescence; aged EPCs are less able to contribute to vascular repair and regeneration and thereby contribute to the increased propensity toward vascular pathology $(5,6)$. Therefore, it is critical to further elucidate the mechanism responsible for the senescence of EPCs in order to provide novel insights into therapeutic neovascularization.

Oxidized low-density lipoprotein (ox-LDL) is taken up by EPCs in a receptor-dependent manner wherein it generates oxidative stress. The level of ox-LDL increases in patients with coronary artery disease or diabetes and serves as an independent predictor for future cardiac events in these patients $(7,8)$. Several studies have shown that ox-LDL functions as an important factor that adversely affects the growth and bioactivity of EPCs by triggering apoptosis, inducing cell ossification and inhibiting endothelialization (9-12). It has also been demonstrated that ox-LDL also accelerated the senescence of EPCs, leading to cellular dysfunction $(13,14)$. Lai and Liu showed that ox-LDL-induced senescence of EPCs was mediated by oxidative stress and the Akt/hTERT pathway (15).

Visfatin was originally cloned in 1994 as a cytokine named pre-B cell colony-enhancing factor (PBEF) from a bone marrow cDNA library based on sequence similarities to cytokines (16). It is now well established that visfatin is expressed in adipose tissue as well as skeletal muscle, liver and immune cells. Visfatin has also been found to be produced within the myocardium localized to cardiomyocytes and fibroblasts, and within the brain localized to neuronal cells, with particularly high expression during ischemia. The widespread distribution of this molecule is indicative of the broad functions of visfatin in both health and disease. Recently, visfatin has been implicated in the pathogenesis of various cardiovascular disorders, such as atherosclerosis and myocardial failure, and in brain ischemia (16). van der Veer et al showed that FK866, an antagonist of visfatin, induced premature senescence in human vascular smooth muscle cells, together with enhanced resistance to oxidative stress (17). However, the effects of viastatin on the ox-LDL-induced senescence of EPCs as well as the underlying mechanism responsible 
for these effects has not yet been explored, to the best of our knowledge. In the present study, we report that visfatin is a senescence-related protein that inhibits the ox-LDL-induced senescence of EPCs by activating sirtuin 1 (SIRT1) through the phosphoinositide-3-kinase (PI3K)/Akt/extracellular signalregulated kinase (ERK) pathway.

\section{Materials and methods}

Cell culture. EPCs were purchased from the American Type Culture Collection (ATCC; Rockville, MD, USA) and cultured in RPMI-1640 supplemented with $10 \%$ fetal bovine serum (FBS) and $100 \mu \mathrm{g} / \mathrm{ml}$ penicillin/streptomycin (all from Invitrogen, Carlsbad, CA, USA).

Ox-LDL preparation. The fresh human plasma was collected from patients at Xiangya Hospital of Central South University (Changsha, China). Human ox-LDL was isolated from fresh human plasma by sequential ultracentrifugation, as previously described (18). Briefly, LDL was isolated by ultracentrifugation at 290,000 x g for $4 \mathrm{~h}$ in a sodium bromide $(\mathrm{NaBr})$ gradient, and the top layer (containing LDL) was collected. To validate the observations made with copper-oxidized LDL, we performed additional experiments using L5, an electronegative and minimally oxidized LDL circulating in patients with hypercholesterolemia.

Telomeric repeat amplification protocol (TRAP) assay. For quantitative analysis of telomerase activity, the TRAP assay, in the which the telomerase reaction product is amplified by a polymerase chain reaction (PCR), was performed using the TeloTAGGG PCR ELISA ${ }^{\text {PLUS }}$ kit (Roche Molecular Biochemicals, Mannheim, Germany) according to the manufacturer's instructions.

Construction of shRNAs. We designed and purchased three different shRNA duplexes of SIRT1 from Gene Pharma (Shanghai, China). The following primers were used: shRNA-1 (SIRT1-homo-1075) sense, 5'-CACCGCAACTATACCCAGA ACATAGTTCAAGAGACTATGTTCTGGGTATAGTTGCTTT TTTG-3' and reverse, 5'-GATCCAAAAAAGCAACTAT ACCCAGAACATAGTCTCTTGAACTATGTTCTGGGTAT AGTTGC-3'; shRNA-2 (SIRT1-homo-1961) sense, 5'-CACCG CTTGATGGTAATCAGTATCTTTCAAGAGAAGATACTG ATTACCATCAAGCTTTTTTG-3' and reverse, 5'-GATCCA AAAAAGCTTGATGGTAATCAGTATCTTCTCTTGAAAG ATACTGATTACCATCAAGC-3'; shRNA-3 (SIRT1-homo2214) sense, 5'-CACCGGAGATGATCAAGAGGCAATTTC AAGAGAATTGCCTCTTGATCATCTCCT TTTTTG-3 and reverse, 5'-GATCCAAAAAAGGAGATGATCAAGA GGCAATTCTCTTGAAATTGCCTCTTGATCATCTCC-3.

Transfection. Twenty-four hours prior to transfection, the cells were seeded in a 6-well culture plate. SIRT1 shRNAs were transfected using Lipofectamine 2000 (Invitrogen) according to the manufacturer's instructions. Twenty-four or forty-eight hours later, the EPCs were collected and subjected to further analysis. Total RNA or protein was extracted from the indicated cells for analysis. The experiment was peformed in triplicate. More than nine wells were treated with the same type of shRNA.
$R N A$ extraction and reverse transcription-quantitative $P C R$ $(R T-q P C R)$. Total RNA was isolated from the cells using TRIzol reagent (Invitrogen) according to the manufacturer's instructions. qPCR was performed using the StepOne Plus sequence detection system (Applied Biosystems, Foster City, CA, USA). To ensure the reproducibility of the results, all the genes were tested in triplicate. The following primers were used: SIRT1 sense (5'-TAGCCTTGTCAGATAAGGAAGGA-3') and antisense (5'-ACAGCTTCACAGTCAACTTTGT-3'); p53 sense (5'-ACTTGTCGCTCTTGAAGCTAC-3') and antisense (5'-GATGCGGAGAATCTTTGGAACA-3'); AKT1 sense (5'-AGCGACGTGGCTATTGTGAAG-3') and antisense (5'-GCCATCATTCTTGAGGAGGAAGT-3'); PI3K/p85 sense (5'-ACACCACGGTTTGGACTATGG-3') and antisense (5'-GGCTACAGTAGTGGGCTTGG-3'); ERK sense (5'-ACACCACGGTTTGGACTATGG-3') and antisense (5'-GGCTACAGTAGTGGGCTTGG-3'); and $\beta$-actin sense, (5'-CATTAAGGAGAAGCTGTGCT-3') and antisense (5'-GTTGAAGGTAGTTTCGTGGA-3'). The cycling conditions were an initial denaturation for $2 \mathrm{~min}$ at $94^{\circ} \mathrm{C}$; followed by 40 cycles of $10 \mathrm{sec}$ at $94^{\circ} \mathrm{C}, 30 \mathrm{sec}$ at $55^{\circ} \mathrm{C}$, and $30 \mathrm{sec}$ at $72^{\circ} \mathrm{C}$.

Western blot analysis. Whole cell extracts were prepared with a cell lysis reagent (Sigma-Aldrich, St.Louis, MO, USA) according to the manufacturer's instructions, and then, the protein was quantified using a BCA assay (Pierce, Rockford, IL, USA). The protein samples were then separated by SDS-PAGE (10\%) and detected by western blot analysis using polyclonal rabbit anti-PI3K p85 antibody (\#4257; Cell Signaling Technology, Danvers, MA, USA, ), rabbit anti-p53 antibody (sc-6243; Santa Cruz Biotechnology, Santa Cruz, CA, USA), mouse anti- $\beta$ galactosidase (Gal) antibody (Cell Signaling Technology, \#2372), rabbit anti-Akt antibody (\#9272; Cell Signaling Technology), rabbit anti-SIRT1 antibody (ab110304; Abcam, Cambridge, UK), rabbit anti-phosphorylated (p-)Akt(Ser473) antibody (ab81283; Abcam), rabbit anti-ERK antibody (\#4695; Cell Signaling Technology), rabbit anti-p-ERK antibody (\#9101; Cell Signaling Technology) and monoclonal mouse antiGAPDH (SC-365062; Santa Cruz Biotechnology) as a control. Goat anti-rabbit IgG (Pierce) secondary antibody conjugated to horseradish peroxidase and ECL detection systems (SuperSignal West Femto; Pierce) were used for detection.

Statistical analysis. Each experiment was repeated at least three times. Data are shown as the means \pm SD and were analyzed using SPSS 18.0. Statistical comparisons between groups were analyzed using the Student's t-test and a two-tailed $\mathrm{p}<0.05$ was considered to indicate a statistically significant difference.

\section{Results}

Ox-LDL induces EPC senescence. To assess the onset of senescence, $\beta$-Gal expression and telomerase activity were detected as biochemical markers of cellular senescence. As shown in Fig. 1A, ox-LDL exposure evidently increased $\beta$-Gal expression. The acceleration of EPC senescence occurred dose dependently, with a maximal effect achieved at a concentration of $4.0 \mathrm{nM}$ ox-LDL.

Cellular senescence is critically influenced by telomerase, which elongates telomeres, thereby counteracting the 

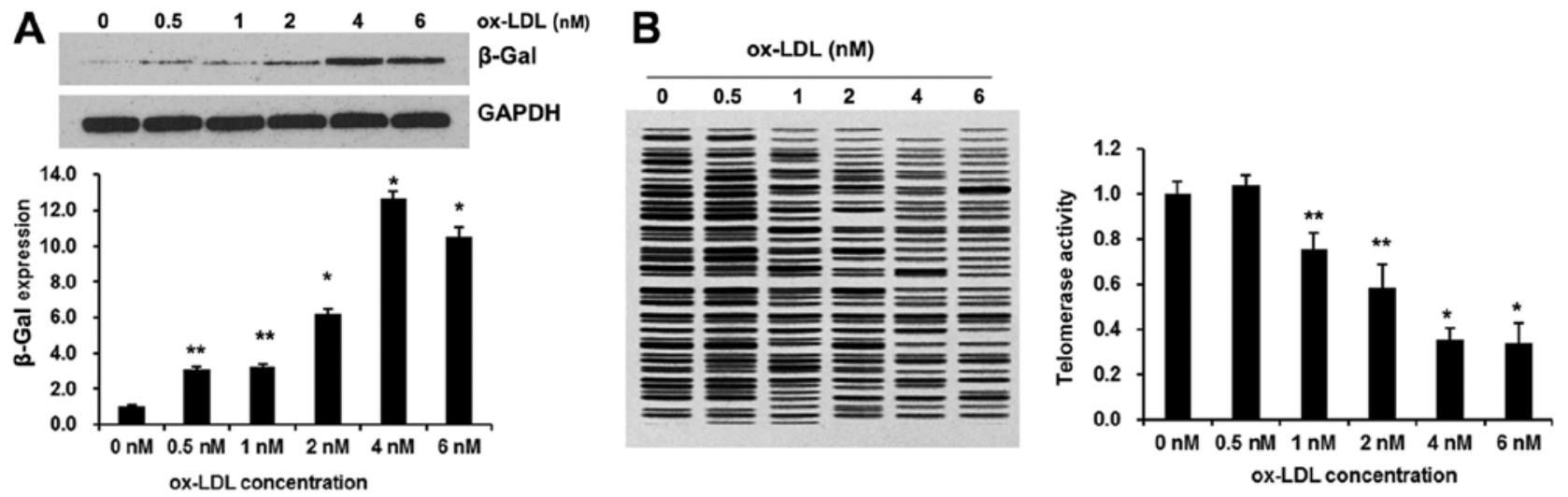

Figure 1. Dose-response relationships between oxidized low-density lipoprotein (ox-LDL) and the senescence of endothelial progenitor cells (EPCs). EPCs were treated with the indicated concentrations of ox-LDL for $24 \mathrm{~h}$. (A) The effects of ox-LDL on $\beta$-galactosidase ( $\beta$-Gal) expression. (B) The effects of ox-LDL on telomerase activity in EPCs. Error bars represent \pm SD and ${ }^{*}$ p $<0.01$ versus. control and $0 \mathrm{nM}$ ox-LDL. ${ }^{* *} \mathrm{p}<0.05$ vs. $0 \mathrm{nM}$ ox-LDL.

A

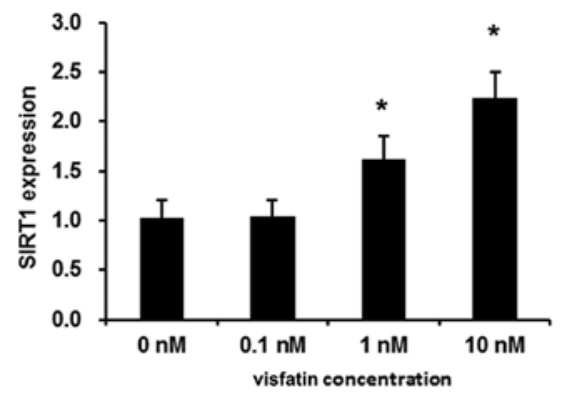

B

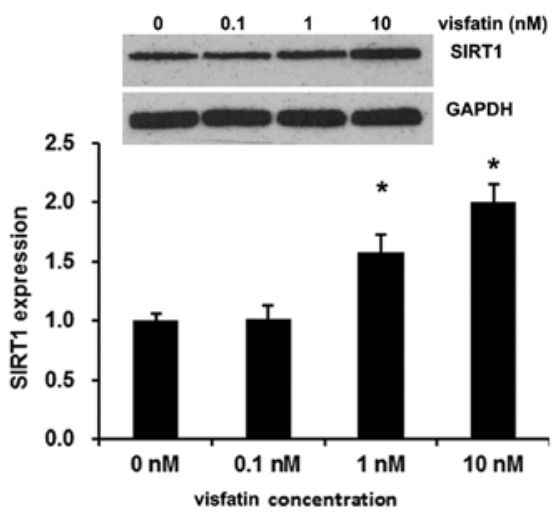

Figure 2. Visfatin affects sirtuin 1 (SIRT1) expression in endothelial progenitor cells (EPCs). EPCs were treated with the indicated concentrations of visfatin for $30 \mathrm{~min}$. (A) RT-qPCR reveals the effects of visfatin on SIRT1 expression. (B) Western blot analysis reveals the effects of visfatin on SIRT1 expression. Error bars represent $\pm \mathrm{SD}$ and ${ }^{*} \mathrm{p}<0.01$ versus. control and $0 \mathrm{nM}$ ox-LDL.

telomere length reduction induced by each cell division. Breitschopf $e t$ al have demonstrated that pro-atherosclerotic factors impair telomerase activity in mature endothelial cells (19). Therefore, we measured telomerase activity using the TeloTAGGG PCR ELISA ${ }^{\text {PLUS }}$ kit. As demonstrated in Fig. 1B, $4.0 \mathrm{nM}$ ox-LDL significantly diminished telomerase activity to approximately $60 \%$. These results clearly demonstrated that 4.0 nM ox-LDL significantly accelerates the senescence of EPCs.

Visfatin induces SIRT1 expression in EPCs. SIRT1 is an anti-aging molecule and SIRT1 expression and activity reduction are involved in aging-associated diseases $(20,21)$. In the present study, exogenetic visfatin evidently increased SIRT1 expression. The acceleration of the mRNA and protein expression of SIRT1 occurred dose dependently, with a maximal effect achieved at a concentration of $10 \mathrm{nM}$ visfatin (Fig. 2).

Visfatin abolishes ox-LDL-induced senescence in EPCs by upregulating SIRTI. Herein, we analyzed the effects of visfatin on SIRT1 and p53 in ox-LDL-stimulated EPCs. Firstly, 3 SIRT1 shRNAs were used to silence SIRT1 expression. As shown in Fig. 3A, shRNA2 effectively blocked the expression of SIRT1 mRNA. Western blot analysis revealed marked reductions of $82.2 \%$ with shRNA2, $72.6 \%$ with shRNA3, and $3.2 \%$ with
shRNA1 in the protein levels of SIRT1 following the transfection of EPCs with SIRT1 shRNAs, compared with the control (untransfected) group and NC (negtive control) group (Fig. 3B) $(\mathrm{p}<0.01)$. Thus, EPCs transfected with SIRT1 shRNA2 were used for subsequent experiments. Next, $10 \mathrm{nM}$ visfatin evidently increased the mRNA (Fig. 3C) and protein (Fig. 3D) expression levels of SIRT1 in ox-LDL-stimulated EPCs, which were reversed by SIRT1 shRNA. Moreover, $10 \mathrm{nM}$ visfatin evidently decreased the mRNA (Fig. 3E) and protein (Fig. 3F) expression levels of p53 in ox-LDL-stimulated EPCs, which were also reversed by SIRT1 shRNA.

We then analyzed the role of visfatin in the ox-LDL-induced senescence of EPCs. The treatment of EPCs with visfatin decreased senescence-associated- $\beta-$ Gal expression (Fig. 4A). Moreover, visfatin evidently reversed the ox-LDL-induced decrease in telomerase activity (Fig. 4B). These results raised the possibility that visfatin may abolish the ox-LDL-induced senescence of EPCs. We then analyzed the role of SIRT1 in the ox-LDL-induced senescence of EPCs. We found that SIRT1-shRNA abolished the effects of visfatin on the ox-LDLinduced senescence of EPCs. In cultured mesangial cells, it has been demonstrated that SIRT1 inhibits oxidative stress-related apoptosis through p53 deacetylation (22). Herein, we found that visfatin evidently decreased p53 expression in EPCs, 

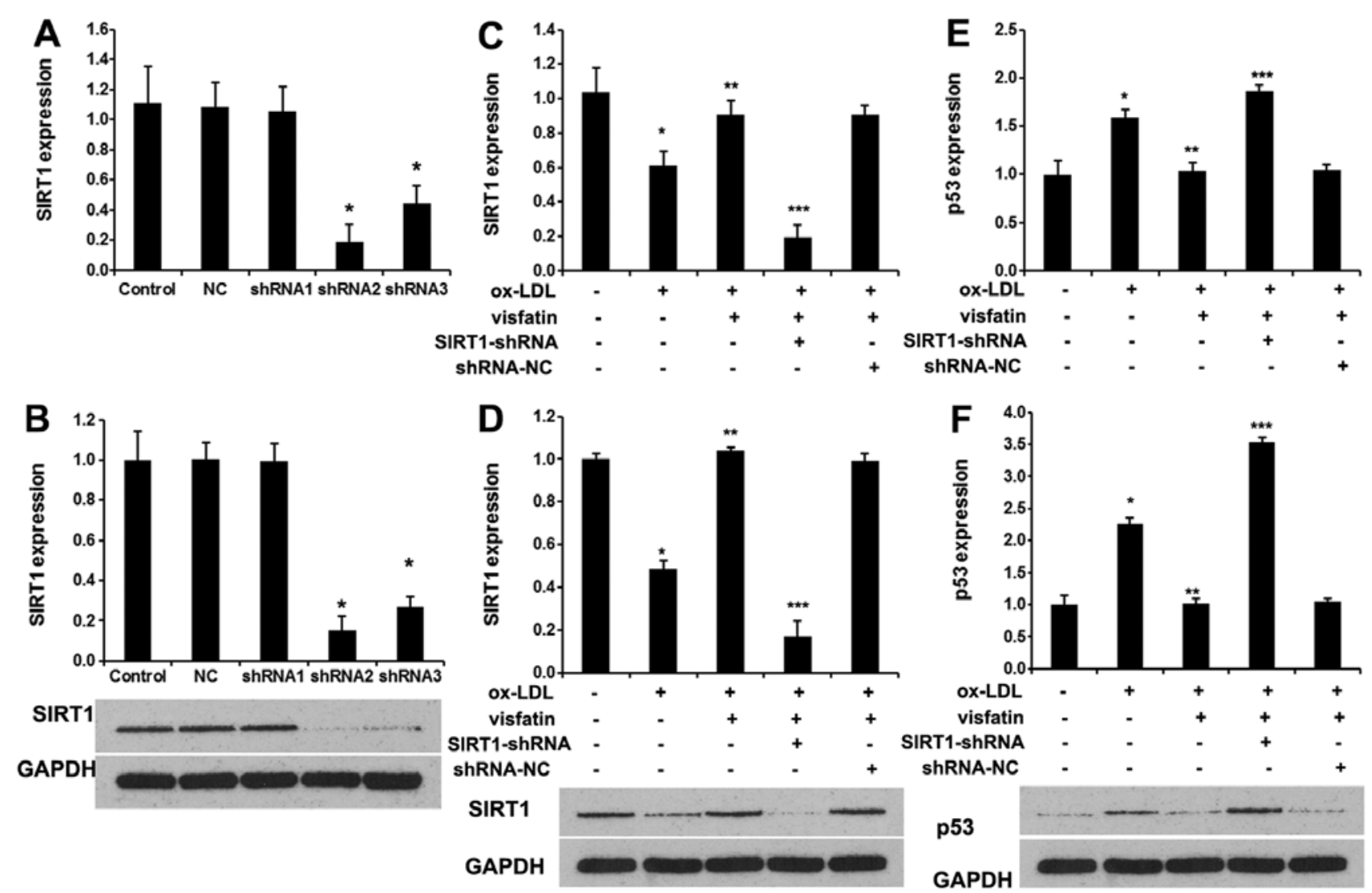

Figure 3. Visfatin affects sirtuin 1 (SIRT1) and p53 expression in oxidized low-density lipoprotein (ox-LDL)-stimulated endothelial progenitor cells (EPCs). EPCs were treated with $4.0 \mathrm{nM}$ ox-LDL, $10 \mathrm{nM}$ visfatin and SIRT1shRNA. (A)RT-qPCR and (B) western blot analysis revealed the effects of SIRT1 shRNAs on SIRT1 expression in EPCs. (C) RT-qPCR and (D) western blot analysis revelaed SIRT1 expression in EPCs. (E) RT-qPCR and (F) western blot analysis revealed p53 expression in EPCs. Error bars represent \pm SD. ${ }^{*} p<0.01$ versus control group. ${ }^{* *} \mathrm{p}<0.01$ vs. ox-LDL treatment group. ${ }^{* * *} \mathrm{p}<0.01$ vs. ox-LDL and visfatin treatment group.
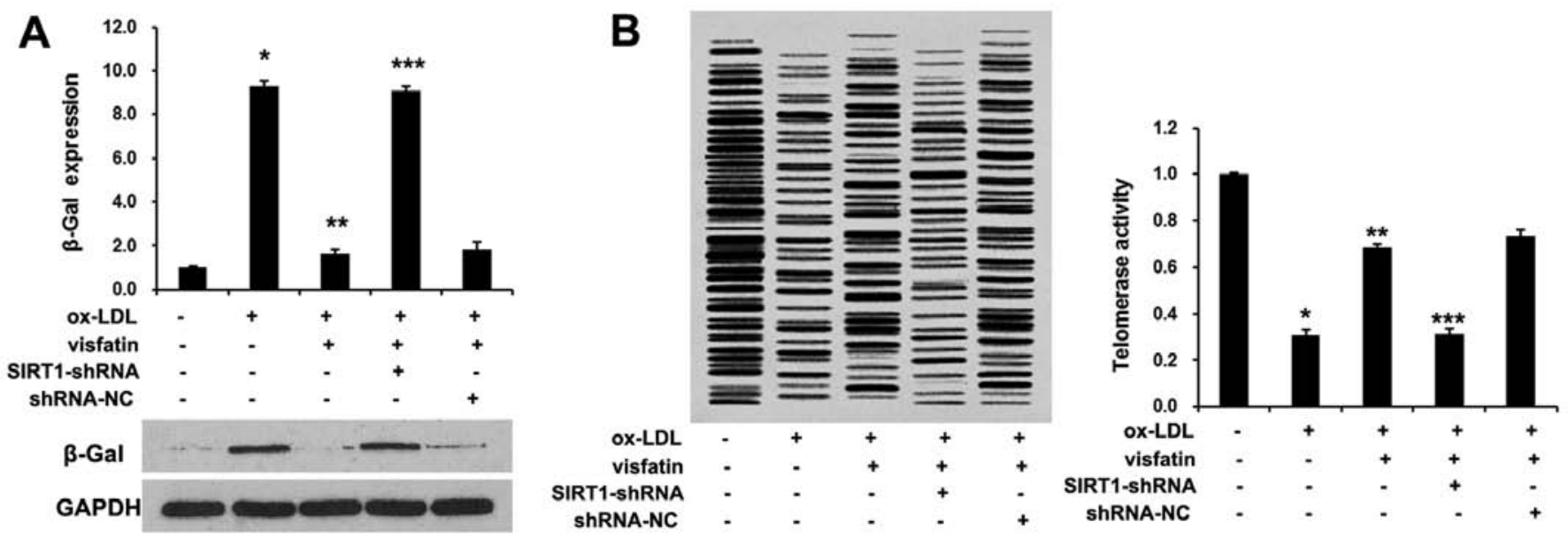

Figure 4. Visfatin abolishes oxidized low-density lipoprotein (ox-LDL)-induced senescence in endothelial progenitor cells (EPCs). EPCs were treated with $4.0 \mathrm{nM}$ ox-LDL, $10 \mathrm{nM}$ visfatin and SIRT1shRNA. EPC senescence was evaluated by (A) $\beta$-galactosidase ( $\beta$-Gal) expression and (B) telomerase activity of EPCs. Error bars represent \pm SD. ${ }^{*} p<0.01$ versus control group. ${ }^{* *} p<0.01$ versus ox-LDL treatment group. ${ }^{* * *} \mathrm{p}<0.01$ versus ox-LDL and visfatin treatment group.

which was reversed by SIRT1-shRNA. In conclusion, these results indicated that visfatin abolished the ox-LDL-induced senescence of EPCs by upregulating SIRT1 expression.

Visfatin regulates SIRT1 expression through the PI3K/Akt/ERK pathway. To examine the role of the PI3K/Akt/ERK pathway in the visfatin-mediated senescence of EPCs, the cells were treated with ox-LDL and then visfatin, finally the inhibi- tors, LY294002, SCH772984. SCH772984 (Active Biochem, Maplewood, NJ, USA) was used to inhibit ERK and LY294002 (Selleck Chemicals, Houston, TX, USA) was used to inhibit PI3K. Consistent with previous findings (18), ox-LDL disrupts the PI3K/Akt signaling pathway by reducing the p-Akt/Akt and p-PI3K/PI3K (p85) ratio in EPCs (Fig. 5), which was reversed by visfatin treatment. Furthermore, LY294002 abolished visfatin-induced PI3K phosphorylation and Akt 

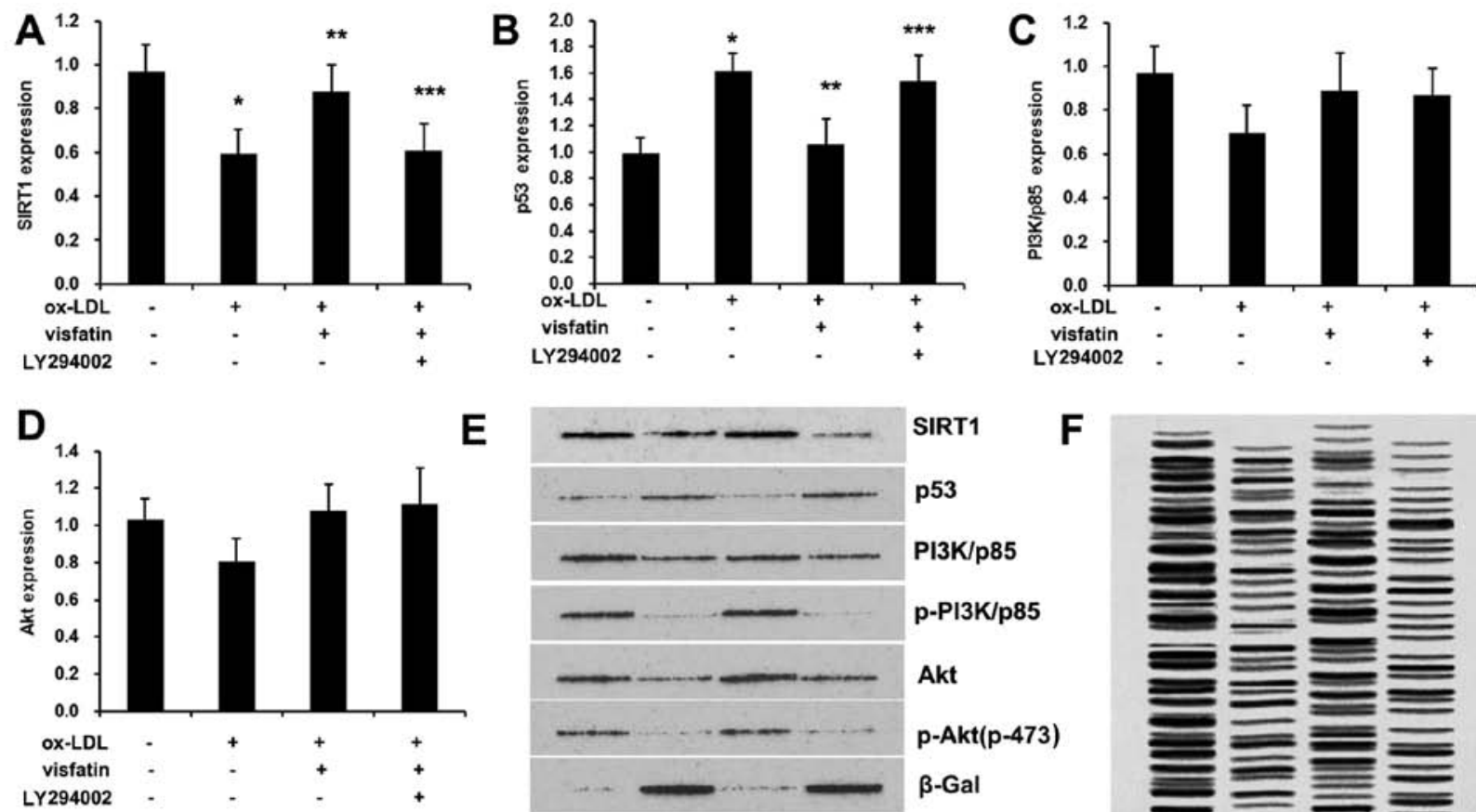

$\mathbf{E}$
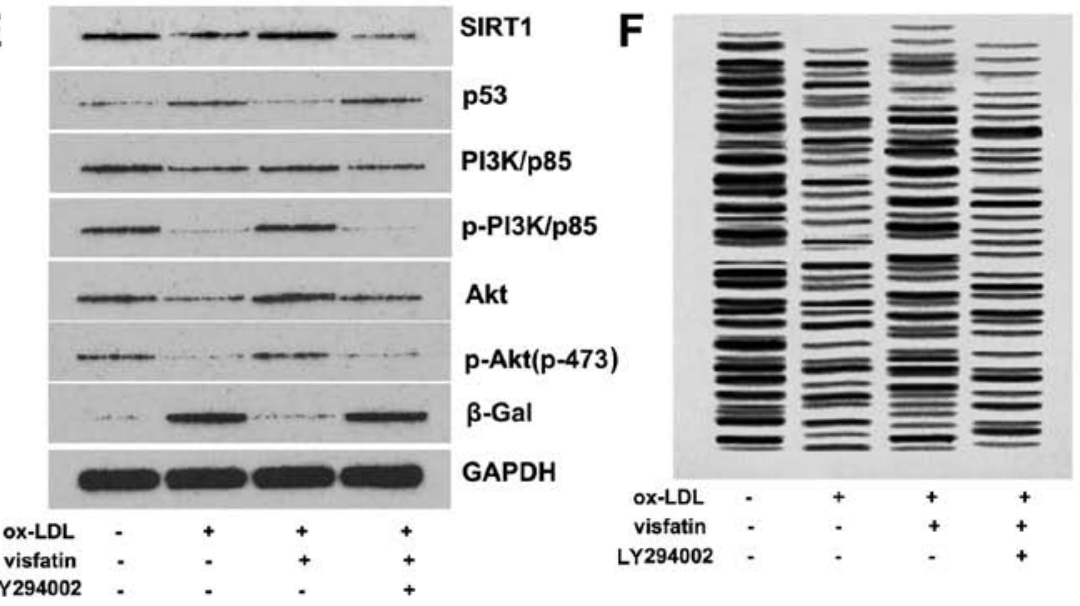

Figure 5. Visfatin regulates sirtuin 1 (SIRT1) expression through the PI3K/Akt pathway. Endothelial progenitor cells (EPCs). were treated with $4.0 \mathrm{nM}$ oxidized low-density lipoprotein (ox-LDL), $10 \mathrm{nM}$ visfatin and PI3K inhibitor (LY294002). RT-qPCR reveals (A) SIRT1 expression, (B) p53 expression, (C) PI3K expression and (D) Akt expression in EPCs. (E) Western blot analysis reveals SIRT1, p53, PI3K/p85, phosphorylated (p-)PI3K/p85, Akt, p-Akt(p-473) and $\beta$-galactosidase ( $\beta$-Gal) expression in EPCs. (F) Telomerase activity of EPCs. Error bars represent \pm SD. ${ }^{*} \mathrm{p}<0.01$ versus control group. ${ }^{* *} \mathrm{p}<0.01$ vs. ox-LDL treatment group. ${ }^{* * *} \mathrm{p}<0.01$ versus ox-LDL and visfatin treatment group.

phosphorylation (Fig. 5E). In addition, LY294002 abrogated the visfatin-induced increase in the expression levels of SIRT1, as well as the visfatin-induced decrease in the expression of p53 (Fig. 5A, B and E), and diminished telomerase activity (Fig. 5F). These results suggested that the PI3K/Akt pathway was regulated by visfatin in order to mediate SIRT1 expression and ox-LDL-induced senescence of EPCs. Moreover, ox-LDL also reduced the p-ERK/ERK ratio in EPCs (Fig. 6D), which was reversed by visfatin treatment. Pre-treatment with SCH772984 abolished the visfatin-induced increase in ERK phosphorylation (Fig. 6D). It should be noted that no significant effects were observed on ERK mRNA expression (Fig. 6C). In addition, SCH772984 abolished the visfatin-induced increase in the expression levels of SIRT1, as well the visfatin-induced decrease in $\mathrm{p} 53$ expression (Fig. 6A, B and D), and diminished telomerase activity (Fig. 5F), suggesting that visfatin-regulated SIRT1 expression and ox-LDL-induced senescence of EPCs involved ERK phosphorylation. In conclusion, visfatin regulates SIRT1 expression in ox-LDL-induced EPC senescence through the PI3K/Akt/ERK pathway.

\section{Discussion}

Previous findings suggest that circulating EPCs serve as a biological marker for vascular function (23) and that the number of EPCs is a significant predictor of future cardiovascular events $(24,25)$. EPCs also play an imporant role in post-ischemic vascular repair $(26,27)$. Ox-LDL, as one of the most important risk factors for cardiovascular disease, has been revealed to exert a detrimental effect on the growth and bioactivity of EPCs by triggering apoptosis, inducing cell ossification and inhibiting endothelialization (9-12). For example, Ji et al showed that ox-LDL significantly decreased the proliferation, migration and adhesion capacity of EPCs, whereas ROS production and NADPH oxidase expression was significantly increased (28). Ox-LDL stimulates p53-dependent activation of pro-apoptotic Bax leading to apoptosis of differentiated EPCs (29). Ox-LDL induced dose- and time-dependent activation of p38 MAPK, which plays a critical role in regulating the numbers and functions of EPCs (30). Previous findings have demonstrated that ox-LDL accelerates the onset of EPC senescence, which may be related to telomerase inactivation, leading to the impairment of proliferative capacity and network formation (13). Consistent with this study (13), the present study found that ox-LDL induces EPC senescence by evidently increasing $\beta$-Gal expression and diminishing telomerase activity.

Visfatin has been implicated in the pathogenesis of various cardiovascular disorders including atherosclerosis and myocardial failure, and in brain ischemia (16,31). Previously, van der Veer et al showed that FK866, an antagonist of visfatin, induced premature senescence in human vascular smooth muscle cells, indicating visfatin is a potential anti-senescence molecule (17). In this study, we found that $10 \mathrm{nM}$ visfatin evidently abolished ox-LDL-induced EPC senescence, thereby providing a novel therapeutic target for the reversal of EPC dysfunction in neovascularization. 

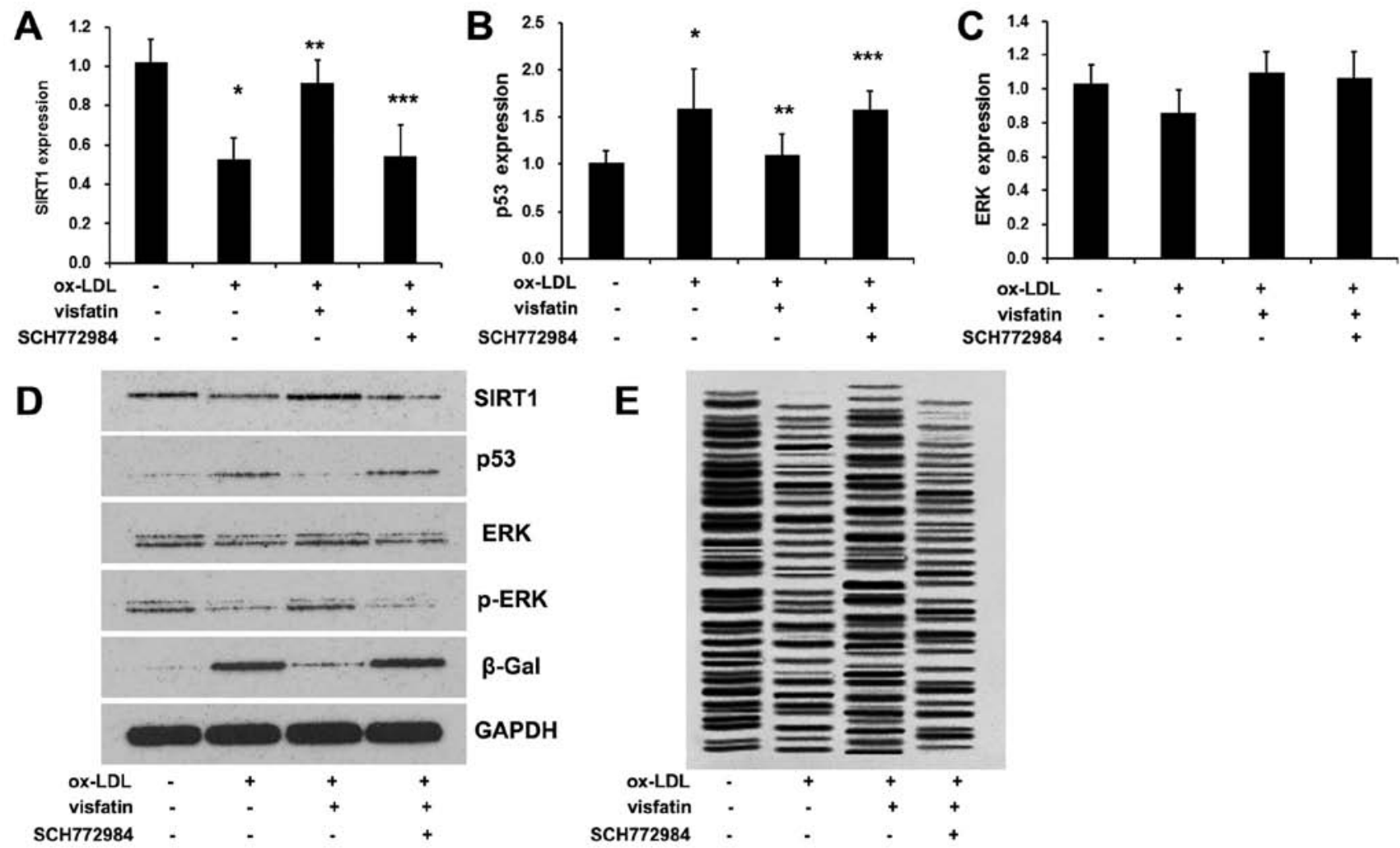

Figure 6. Visfatin regulates sirtuin 1 (SIRT1) expression through the ERK pathway. Endothelial progenitor cells (EPCs) were treated with $4.0 \mathrm{nM}$ oxidized low-density lipoprotein (ox-LDL), $10 \mathrm{nM}$ visfatin and ERK inhibitor (SCH772984). RT-qPCR reveals (A) SIRT1 expression, (B) p53 expression and (C) ERK expression in EPCs. (D) Western blot analysis reveals SIRT1, p53, ERK, phosphorylated (p-)ERK and $\beta$-galactosidase ( $\beta$-Gal) expression in EPCs. (E) Telomerase activity of EPCs. Error bars represent \pm SD. ${ }^{*}$ p $<0.01$ versus control group. ${ }^{* *} \mathrm{p}<0.01$ versus ox-LDL treatment group. ${ }^{* * *} \mathrm{p}<0.01$ versus ox-LDL and visfatin treatment group.

The family of sirtuin proteins are a class of $\mathrm{NAD}^{+}$-dependent protein deacetylases which are proteins regulating key cellular processes including cell cycle regulation, apoptosis, metabolic regulation and inflammation (20). SIRT1 is reported to be a regulator of ageing and its increased expression or activation prolonged lifespan in lower forms of animals (32). For example, Braidy et al demonstrated that SIRT1 expression increases with age, concurrently with increased acetylated p53 levels in all brain regions investigated (21). Kume et al showed that SIRT1 attenuates oxidative stress-induced mesangial cell apoptosis through p53 deacetylation (22). A previous study showed that SIRT1 is a critical modulator of EPC dysfunction during alterations of glucose metabolism (33), indicating that the modulation of SIRT1 activity and/or expression may provide an opportunity for counteracting the impairment of EPC levels and functionality associated with the ageing of EPCs. Moreover, dimethylarginine dimethylaminohydrolase 2 (DDAH)/asymmetric dimethylarginine (ADMA) is involved in the accelerated senescence of EPCs in diabetes, which is associated with the activation of SIRT1 (34). In this study, we found that downregulated SIRT1 expression is associated with ox-LDL-induced EPC senescence. We further analyzed the association between visfatin and SIRT1. We found that visfatin significantly upregulated SIRT1 expression and silenced SIRT1 evidently abolished the inhibition of senescence in EPCs and the suppression of p53 expression induced by visfatin. In conclusion, these results indicated that visfatin abolished the ox-LDL-induced senescence of EPCs by upregulating SIRT1 expression.
The PI3K/Akt signaling pathway is critical for the function and survival of EPCs (35), based largely on the downstream activation of endothelial nitric oxide synthase (eNOS) and subsequent production of NO (9,36). Imanishi et al (11) reported that ox-LDL impairs EPC function through effects on Akt. Imanishi et al showed that the ox-LDL effects on Akt within EPCs are mediated indirectly, by an upstream disruption of PI3K, specifically via nitrosylation of the p85 subunit and subsequent dissociation of the p85 and p110 subunits (13). In the present study, LY294002, a PI3K inhibitor, significantly reversed the inhibition of ox-LDL-induced senescence in EPCs as well as SIRT1 expression induced by visfatin, indicating that the PI3K/Akt signaling pathway was regulated by visfatin in order to mediate SIRT1 expression and ox-LDL-induced senescence of EPCs. The ERK pathway is often associated with SIRT1 expression in various types of cell (37). In the present study, we further analyzed whether the ERK signaling pathway is also important in ox-LDL-induced EPC senescence. We found that ox-LDL induced a decrease in the p-ERK/ERK ratio, which was reversed by visfatin. SCH772984, a selective inhibitor of ERK, significantly reduced the inhibition of ox-LDL-induced EPC senescence and the increase in SIRT1 expression induced by visfatin.

In conclusion, for the first time to the best of our knowledge, we demonstrate the anti-senescence effects of visfatin in ox-LDL-stimulated EPCs, and show that visfatin markedly attenuates ox-LDL-induced EPC senescence by upregulating SIRT1 expression through the PI3K/Akt/ERK pathway, 
providing a novel therapeutic strategies for the reversal of EPC dysfunction.

\section{Acknowledgements}

The present study was supported by the National Natural Science Foundation of China (no. 81300204, 81302850, 81301106), the Hunan Provincial Natural Science Foundation of China (2015JJ4056) and the Changsha Science and Technology Key Project (K1406004-31,K1308032-31).

\section{References}

1. Khoo CP, Pozzilli P and Alison MR: Endothelial progenitor cells and their potential therapeutic applications. Regen Med 3: 863-876, 2008

2. Fadini GP, Losordo D and Dimmeler S: Critical reevaluation of endothelial progenitor cell phenotypes for therapeutic and diagnostic use. Circ Res 110: 624-637, 2012.

3. Yang C, Zhang ZH, Li ZJ, Yang RC, Qian GQ and Han ZC: Enhancement of neovascularization with cord blood CD133 cell-derived endothelial progenitor cell transplantation. Thromb Haemost 91: 1202-1212, 2004.

4. Ott I, Keller U, Knoedler M, Götze KS, Doss K, Fischer P, Urlbauer K, Debus G, von Bubnoff N, Rudelius M, et al: Endothelial-like cells expanded from CD34 blood cells improve left ventricular function after experimental myocardial infarction. FASEB J 19: 992-994, 2005.

5. Toda N: Age-related changes in endothelial function and blood flow regulation. Pharmacol Ther 133: 159-176, 2012.

6. Loomans CJ, de Koning EJ, Staal FJ, Rookmaaker MB Verseyden C, de Boer HC, Verhaar MC, Braam B, Rabelink TJ and van Zonneveld AJ: Endothelial progenitor cell dysfunction: a novel concept in the pathogenesis of vascular complications of type 1 diabetes. Diabetes 53: 195-199, 2004.

7. Shimada K, Mokuno H, Matsunaga E, Miyazaki T, Sumiyoshi K, Miyauchi K and Daida H: Circulating oxidized low-density lipoprotein is an independent predictor for cardiac event in patients with coronary artery disease. Atherosclerosis 174: 343-347, 2004

8. Shimada K, Mokuno H, Matsunaga E, Miyazaki T, Sumiyoshi K, Kume A, Miyauchi K and Daida $\mathrm{H}$ : Predictive value of circulating oxidized LDL for cardiac events in type 2 diabetic patients with coronary artery disease. Diabetes Care 27: 843-844, 2004.

9. Ma FX, Zhou B, Chen Z, Ren Q, Lu SH, Sawamura T and Han ZC: Oxidized low density lipoprotein impairs endothelial progenitor cells by regulation of endothelial nitric oxide synthase. J Lipid Res 47: 1227-1237, 2006.

10. Rosso A, Balsamo A, Gambino R, Dentelli P, Falcioni R, Cassader M, Pegoraro L, Pagano G and Brizzi MF: p53 mediates the accelerated onset of senescence of endothelial progenitor cells in diabetes. J Biol Chem 281: 4339-4347, 2006.

11. Imanishi T, Hano T, Matsuo Y and Nishio I: Oxidized low-density lipoprotein inhibits vascular endothelial growth factor-induced endothelial progenitor cell differentiation. Clin Exp Pharmacol Physiol 30: 665-670, 2003

12. Liu L, Liu ZZ, Chen H, Zhang GJ, Kong YH and Kang XX: Oxidized low-density lipoprotein and $\beta$-glycerophosphate synergistically induce endothelial progenitor cell ossification. Acta Pharmacol Sin 32: 1491-1497, 2011.

13. Imanishi T, Hano T, Sawamura $\mathrm{T}$ and Nishio I: Oxidized low-density lipoprotein induces endothelial progenitor cell senescence, leading to cellular dysfunction. Clin Exp Pharmacol Physiol 31: 407-413, 2004.

14. Hill JM, Zalos G, Halcox JP, Schenke WH, Waclawiw MA, Quyyumi AA and Finkel T: Circulating endothelial progenitor cells, vascular function, and cardiovascular risk. N Engl J Med 348: 593-600, 2003.

15. Lai P and Liu Y: Angelica sinensis polysaccharides inhibit endothelial progenitor cell senescence through the reduction of oxidative stress and activation of the Akt/hTERT pathway. Pharm Biol 53: 1842-1849, 2015.

16. Dahl TB, Holm S, Aukrust $P$ and Halvorsen B: Visfatin/NAMPT: a multifaceted molecule with diverse roles in physiology and pathophysiology. Annu Rev Nutr 32: 229-243, 2012.

17. van der Veer E, Ho C, O'Neil C, Barbosa N, Scott R, Cregan SP and Pickering JG: Extension of human cell lifespan by nicotinamide phosphoribosyltransferase. J Biol Chem 282: 10841-10845, 2007.
18. Tie G, Yan J, Yang Y, Park BD, Messina JA, Raffai RL, Nowicki PT and Messina LM: Oxidized low-density lipoprotein induces apoptosis in endothelial progenitor cells by inactivating the phosphoinositide 3-kinase/Akt pathway. J Vasc Res 47: 519-530, 2010.

19. Breitschopf K, Zeiher AM and Dimmeler S: Pro-atherogenic factors induce telomerase inactivation in endothelial cells through an Akt-dependent mechanism. FEBS Lett 493: 21-25, 2001.

20. Kume S, Kitada M, Kanasaki K, Maegawa H and Koya D: Anti-aging molecule, Sirt1: a novel therapeutic target for diabetic nephropathy. Arch Pharm Res 36: 230-236, 2013.

21. Braidy N, Poljak A, Grant R, Jayasena T, Mansour H, Chan-Ling T, Smythe G, Sachdev P and Guillemin GJ: Differential expression of sirtuins in the aging rat brain. Front Cell Neurosci 9: 167, 2015.

22. Kume S, Haneda M, Kanasaki K, Sugimoto T, Araki S, Isono M, Isshiki K, Uzu T, Kashiwagi A and Koya D: Silent information regulator 2 (SIRT1) attenuates oxidative stress-induced mesangial cell apoptosis via p53 deacetylation. Free Radic Biol Med 40: 2175-2182, 2006.

23. Bakogiannis C, Tousoulis D, Androulakis E, Briasoulis A, Papageorgiou N, Vogiatzi G, Kampoli AM, Charakida M, Siasos G, Latsios G, et al: Circulating endothelial progenitor cells as biomarkers for prediction of cardiovascular outcomes. Curr Med Chem 19: 2597-2604, 2012.

24. Reynolds JA, Robertson AC, Bruce IN and Alexander MY: Improving cardiovascular outcomes in rheumatic diseases: therapeutic potential of circulating endothelial progenitor cells. Pharmacol Ther 142: 231-243, 2014.

25. Werner N, Kosiol S, Schiegl T, Ahlers P, Walenta K, Link A, Böhm M and Nickenig G: Circulating endothelial progenitor cells and cardiovascular outcomes. N Engl J Med 353: 999-1007, 2005.

26. Takahashi T, Kalka C, Masuda H, Chen D, Silver M, Kearney M, Magner M, Isner JM and Asahara T: Ischemia- and cytokineinduced mobilization of bone marrow-derived endothelial progenitor cells for neovascularization. Nat Med 5: 434-438, 1999.

27. Aicher A, Rentsch M, Sasaki K, Ellwart JW, Fändrich F, Siebert R, Cooke JP, Dimmeler S and Heeschen C: Nonbone marrow-derived circulating progenitor cells contribute to postnatal neovascularization following tissue ischemia. Circ Res 100: 581-589, 2007.

28. Ji KT, Qian L, Nan JL, Xue YJ, Zhang SQ, Wang GQ, Yin RP, Zhu YJ, Wang LP, Ma J, et al: Ox-LDL induces dysfunction of endothelial progenitor cells via activation of NF- $\kappa$ B. BioMed Res Int 2015: 175291, 2015.

29. Cheng J, Cui R, Chen $\mathrm{CH}$ and Du J: Oxidized low-density lipoprotein stimulates p53-dependent activation of proapoptotic Bax leading to apoptosis of differentiated endothelial progenitor cells. Endocrinology 148: 2085-2094, 2007.

30. Wu Y, Wang Q, Cheng L, Wang J and Lu G: Effect of oxidized low-density lipoprotein on survival and function of endothelial progenitor cell mediated by p38 signal pathway. J Cardiovasc Pharmacol 53: 151-156, 2009.

31. Revollo JR, Körner A, Mills KF, Satoh A, Wang T, Garten A, Dasgupta B, Sasaki Y, Wolberger C, Townsend RR, et al: Nampt/ $\mathrm{PBEF} /$ visfatin regulates insulin secretion in beta cells as a systemic NAD biosynthetic enzyme. Cell Metab 6: 363-375, 2007.

32. Poulose $\mathrm{N}$ and Raju R: Sirtuin regulation in aging and injury. Biochim Biophys Acta 1852: 2442-2455, 2015.

33. Balestrieri ML, Rienzo M, Felice F, Rossiello R, Grimaldi V, Milone L, Casamassimi A, Servillo L, Farzati B, Giovane A and Napoli C: High glucose downregulates endothelial progenitor cell number via SIRT1. Biochim Biophys Acta 1784: 936-945, 2008.

34. Yuan Q, Hu CP, Gong ZC, Bai YP, Liu SY, Li YJ and Jiang JL: Accelerated onset of senescence of endothelial progenitor cells in patients with type 2 diabetes mellitus: role of dimethylarginine dimethylaminohydrolase 2 and asymmetric dimethylarginine. Biochem Biophys Res Commun 458: 869-876, 2015.

35. Ackah E, Yu J, Zoellner S, Iwakiri Y, Skurk C, Shibata R, Ouchi N, Easton RM, Galasso G, Birnbaum MJ, et al: Akt1/protein kinase Balpha is critical for ischemic and VEGF-mediated angiogenesis. J Clin Invest 115: 2119-2127, 2005.

36. Lefevre J, Michaud SE, Haddad P, Dussault S, Ménard C, Groleau J, Turgeon J and Rivard A: Moderate consumption of red wine (cabernet sauvignon) improves ischemia-induced neovascularization in ApoE-deficient mice: effect on endothelial progenitor cells and nitric oxide. FASEB J 21: 3845-3852, 2007.

37. Chou WW, Chen KC, Wang YS, Wang JY, Liang CL and Juo SH: The role of SIRT1/AKT/ERK pathway in ultraviolet B induced damage on human retinal pigment epithelial cells. Toxicol In Vitro 27: 1728-1736, 2013. 